\title{
LOCATIONAL PLANNING FOR EMERGENCY MANAGEMENT AND RESPONSE: AN ARTIFICIAL INTELLIGENCE APPROACH
}

\author{
YORGOS N. PHOTIS \& GEORGE GREKOUSIS \\ Department of Planning and Regional Development, University of Thessaly, Greece.
}

\begin{abstract}
The efficiency of emergency service systems is measured in terms of their ability to deploy units and personnel in a timely and effective manner upon an event's occurrence. When dealing with public sector institutions, this reflects the significance for state or local officials to determine the optimal locations for emergency stations and vehicles. The typical methodology to deal with such a task is through the application of the appropriate location-allocation model. In such a case, however, the spatial distribution of demand although stochastic in nature and layout, when aggregated at the appropriate level, appears to be spatially structured or semistructured. Aiming to exploit the above incentive, a different approach will be examined in this paper. The spatial tracing and location analysis of emergency incidents is achieved through the utilisation of an Artificial Neural Network (ANN). More specifically, the ANN provides the basis for a spatiotemporal clustering of demand, definition of the relevant centres, formulation of possible future states of the system and finally, definition of locational strategies for the improvement of the provided services. The proposed methodological approach is applied to Athens Metropolitan Area and the adopted dataset constitutes of the incidents that were reported and confronted by the city's Fire Department during the year 2008.
\end{abstract}

Keywords: Emergency planning, fuzzy logic, neural networks, spatiotemporal location analysis.

\section{INTRODUCTION}

The main objective during the confrontation of locational planning problems is the location of facilities or service centres and the simultaneous spatial allocation of demand to them. The problems in question are deterministic, when the critical problem parameters, namely, the spatial distribution of demand, the distances from demand points to the candidate centres and the capacity of facilities, are predefined and constant. In the case that, the values of any of these parameters are allowed to change over time, the corresponding phenomena are considered stochastic. Such spatial problems can be approached via location-allocation models and are solved by examination of a set of alternative location patterns [1]. The aim of these models is to minimise objective functions that usually express some form of cost.

Demand is depicted spatially as a point pattern whose analysis in the case of stochastic problem settings is of decisive importance for its appropriate allocation to supply sites or service centres. The points constituting such a pattern may or may not share equal weights, characterised in this respect as constant or variable over time and their spatial distribution can be either random or following a clustered or regular structure.

For the formulation of robust solutions to location-allocation models, three alternative methods can be utilised: exact analytical numerical solutions or programming techniques and heuristic algorithms and meta-heuristic algorithms [2]. Research during the last decades has revealed many weaknesses in traditional methods of solving location-allocation problems, particularly with respect to the specification of the algorithm's initial solution and the selection of the appropriate objective function [3]. The main advantage of programming techniques is that they always lead to an optimal solution [4]. The basic disadvantage of these algorithms still remains the fact that they cannot 'guarantee' that they will converge to a global and not a local optimal solution. 
During the first decades of the 1990s, meta-heuristics, a new type of algorithms was introduced, based mainly on techniques of artificial intelligence. According to Haykin [5], artificial intelligence is: ' $a$ sector of computer science, which deals with the study and creation of information systems which contain some type of intelligence'. Artificial intelligence is thus composed of four separate modelling technologies: Expert Systems, Fuzzy Logic, Neural Networks and Genetic Algorithms. Applications of such models are mentioned by Guerrero et al. [6], for location allocation models in continuous space and by Wilsons et al. [7], who used expert systems to determine regions of high criminality. In 1996, Houck et al. [8], developed a genetic algorithm for the Weber problem, while in 1997 Gen et al. [9], applied a similar approach to deal with the Capacited Location Problem. Finally, Zhou, Gen et al. [10], in 2002 proposed a genetic algorithm for the balanced allocation of customers to multiple distribution centres in a supply chain network. These papers reveal the progressive adaptation of artificial intelligence methods and techniques to the successive stages of location planning and modelling process. Furthermore, the integration of GIS with artificial intelligence leads to the development of powerful tools for decision-making with high levels of efficiency [11-15]. With this in mind, the present paper develops neural networks and fuzzy logic for predicting spatial demand patterns and locating supply centres in locational planning problems.

In this paper, we analyse emergency incidents that diachronically occur during successive time periods, and through the identification of their spatiotemporal footprint, we predict their pattern's future evolution. Our goal is to take action on the planning, management and prevention levels. The proposed analytical methodology is based on both GIS functionality and artificial intelligence techniques, such as neural networks and fuzzy logic.

More specifically, in the proposed approach, each location problem is dealt with at three distinct stages. First, the spatial point pattern of demand is analysed over time using the Nearest Neighbour Analysis method. Second, the approach provides the ability to predict, by means of neural networks, how the pattern of demand will develop. Finally, it locates supplying centres and allocates demand to them by the implementation of fuzzy logic.

The application section of our work, deals with the location of the Fire Department's vehicles (fire engines) in the Metropolitan Area of Athens, Greece. Demand prediction, based on diachronic data, allows fire engines to be sited in locations which will minimise distances from the set of expected incidents. Consequently, given that in locational planning the locational choices made are generally judged by the 'quality' of the decision-making process, which generated those choices [16], improved problem analysis will lead to better locational choices and thus better locational patterns.

Diachronic data are analysed and processed by the neural network, which interprets the spatiotemporal pattern of demand and approximates the predicted Cartesian coordinates of future events. In the following stage of the process, the fire engines are optimally located and the allocation of demand to them is accomplished through the utilisation of fuzzy logic and more specifically the Fuzzy C-means algorithm. Finally, in an attempt to assess the effectiveness of the proposed methodology, the results, in terms of predicted event locations are compared with the analogous pragmatic data of supply and demand.

\section{LOCATIONAL PLANNING PROBLEMS}

In general, locational planning problems deal with the spatial organisation of services, which respectively require the location of centres and the allocation of the demand to them, according to specific constraints. In principle, we can differentiate the constraints into two major categories: The first one, which is termed distance-oriented, deals with the maximum time-distance that the population has to travel to get to its centre. The second, which is termed demand-oriented, refers to the minimum and maximum number of demand, which justifies the existence of a certain service centre. In this paper, 
of interest is the second constraint category, which leads to one of the most critical issues in any locational planning strategy or policy action, namely, the assessment of centre utilisation.

More specifically, in most empirical locational planning applications, the degree to which investments for the location of service centres will be finally justified by their future utilisation has yet to be satisfactorily answered. Furthermore, location analysts are greatly interested in the avoidance of mistakes stemming from the absence or inadequate estimation of the critical planning parameters, such as the size and the composition of the population to be served and the way they respond to changing conditions in the problem environment [17].

\subsection{Objectives of locational problems}

The objective of a location allocation model is to find the optimal location of $\mathbf{P}$ centres and to allocate demand $\mathbf{W}$ in the nearest centres. Thus, objectives are determined that depend on the location of centres and then algorithms are defined for finding the optimal or near optimal solution [2].

Location models fall into two types, deterministic and stochastic. In the deterministic type, data are predetermined and do not depend on time. However, most location problems are dynamic [18]. The dynamic nature of problems is dealt with stochastic models in which multiple time periods are contained [19]. These periods facilitate the detection of differences in the average value of demand, the recognition of differences among spatial point patterns and the estimation of future levels of demand [1]. This periodical variability in demand suggests that the optimal solution is not to be found having fixed centres. A classic example of a stochastic problem is the location of ambulances in a city. Future calls for help from ambulances are not known in advance. However, their prediction is of decisive importance so that demand is dealt with in the best possible way [20].

A basic condition for the prediction constitutes the analysis of spatial point pattern of demand through which it is possible to comprehend the structure and to determine the tendencies of a spatial phenomenon.

\subsection{Spatial point pattern analysis}

During the first decades of the 20th century, the analysis of a distribution of points to decide to which morphological type of spatial pattern belongs was considered a complicated and challenging problem.

However, from 1960s onwards, new methods and techniques were introduced as adequate means of testing and interpreting real-world phenomena summarising the general properties of patterns. First, Dacey [21] and later Rogers [22] dealt with the adoption of mathematical models capable of describing and mathematically expressing spatial distributions through a process-to-pattern approach.

Each spatial pattern in certain space and time is considered the result of an earlier process evolving in broader space and time limits. The basic spatial processes that are expressed through the corresponding point patterns are random, attractive and competitive. These lead, respectively, to random, clustered and dispersed point patterns. Nowadays, there are many available descriptive statistics for pattern analysis. The two most widely applied point pattern analysis techniques are: quadrat analysis and nearest neighbour analysis [23].

In this section, the nearest neighbour method will be discussed and illustrated. According to it, distances from every point to its nearest neighbour are calculated, summarised and their mean value is calculated. Then, it is compared to the corresponding mean expected distance of a spatial pattern of the same size, which would result from a random process (Poisson distribution). 
More analytically, as Rogers [22] showed in 1969, if a circle of diameter $\mathrm{d} \alpha$ is considered, then the probability that a point exists in distance $\mathrm{d} \alpha$ (expected distance) follows normal distribution with a mean value given by the eqn (1):

$$
d a=1 / 2 \sqrt{n / A},
$$

where $n$ denotes the number of points in the distribution and $A$ represents the area under study. The observed mean value of distances from every point to each nearest neighbour is calculated according to the eqn (2):

$$
d_{p}=1 / n \sum_{i=1}^{n} d_{i},
$$

where $d_{i}$ denotes the distance from every point $i$ to its nearest neighbour.

This is a sampling value of a theoretical normal distribution. This distribution is the sampling distribution of all likely $d_{p}$. Statistical tables of normal distribution allow us to check how much the observed value $d_{p}$ coincides with the theoretical $d_{a}$. The divergences from random spatial process are determined through the indicator $\mathrm{R}=\mathrm{d}_{\mathrm{p}} / \mathrm{d}_{\mathrm{a}}$ [24]. By means of this indicator, a scale is created. This scale starts with a clustered pattern and progresses through a random pattern to end in a uniform spatial pattern. Thus, if $\mathrm{R}<1$, then the pattern has a tendency to cluster, while, if $\mathrm{R}>1$, then it has the tendency to dispersion. Finally, if $\mathrm{R}=1$, the pattern is random.

Furthermore, $\sigma_{\mathrm{d} \alpha}$, the standard deviation of $\mathrm{d}_{\mathrm{a}}$ is needed, which is given by eqn (3):

$$
\sigma_{d a}=0,2616 / \sqrt{n^{2} / A} \text {. }
$$

Finally, Z value is calculated, from eqn (4), and is compared to the value that results from statistical tables of normal distribution for the desired level of confidence.

$$
Z=\frac{d_{\pi}-d_{a}}{\sigma_{d a}}
$$

The comparison aims at checking the null hypothesis $\mathrm{H}_{0}$ and the hypothesis $\mathrm{H}_{1}$. The probability that the criterion value appears is calculated. If the probability is smaller than the level of confidence, then the null hypothesis $\mathrm{H}_{0}$ is rejected and $\mathrm{H}_{1}$ is accepted. If probability is greater than the level of confidence, then the null hypothesis $\mathrm{H}_{0}$ can neither be rejected nor accepted [25].

\subsection{Artificial intelligence}

Methods and techniques that incorporate and simulate basic characteristics of human thought to solve practical problems are part of the science of artificial intelligence. Expert systems, neural networks, genetic algorithms and fuzzy logic are methods and techniques of artificial intelligence and imitate the way that a human learns, creates symbols, recognises and represents elements of reality. In the present paper, the techniques of neural networks and fuzzy logic are embedded in the proposed methodology.

\subsubsection{Neural networks}

Neural networks are systems that incorporate and simulate basic characteristics of human thought to solve practical problems. The goal of a neural network is to recognise the mathematical patterns among data sets [26, 27]. According to Haykin [4], a neural net is a parallel processor system that stores and analyses knowledge like a human brain. Another definition is that a neural net is a simulation of a human brain that learns to recognise mathematical patterns among the data. 
The process of setting up the architecture of a neural network is complicated. For each network, a set of parameters, such as the number of hidden layers, the number of neurons per layer, the learning rate, the epochs of iteration and the transfer functions needs to be regulated. The appropriate setting of these parameters is of crucial importance, for the final precision and acceptance of network depends on them.

\subsubsection{Fuzzy logic}

Fuzzy logic theory was developed to handle problems that do not have clear boundaries or cases in which ambiguity is inherent. It can be regarded as a generalisation of the classic theory of sets and as a generalisation of dual logic. Classic mathematical dual logic has a tendency to model the real world so that everything fits in it. For Aristotelian logic, that is classic: everything is characterised with a 'yes' or 'no'. Anything that cannot be characterised as such is abandoned or transformed to fit into this binary selection. In the real world, on the other hand, there are many ambiguous cases; dual logic cannot handle these problems. Lofti Zadeh [28] introduced a new logic, the fuzzy logic, that can handle both binary and ambiguous cases.

According to Zadeh, fuzzy logic is the process of making calculations with words. The ability to develop models built on linguistic terms and based on a common feeling or empirical rules is the core of fuzzy logic theory. Fuzzy logic is part of artificial intelligence, which is a set of methods for analysing and representing knowledge similar to the human brain. Fuzzy logic is ideal to achieve this objective, as it provides a method to translate the natural language based on expressions of knowledge and common sense into a precise mathematic language. It is a technology that gives computers the possibility of thinking and of making decisions that simulate humans. [29, 30].

One of the most efficient and well-known algorithms in fuzzy classification is Fuzzy C-means. It analyses input elements, seeks relations among them and finally classifies them. Each class has a cluster centre, which portrays the value of a typical object of that class. The remaining objects are not classified in only one class. Instead, a membership value is calculated expressing the degree that each object belongs to each class.

\section{METHODOLOGICAL FRAMEWORK}

The main aim of the proposed methodology is twofold. First, the approximation of the geographical coordinates of incidents that will hypothetically occur in time $\mathbf{t}+\mathbf{1}$, in the study region, when diachronic data are available until time $\mathbf{t}$ (Graph 1). Second, the optimal allocation of the predicted demand points to a specific number of service centres, which in a locational planning perspective should coincide with the optimal service arrangement for problem at hand.

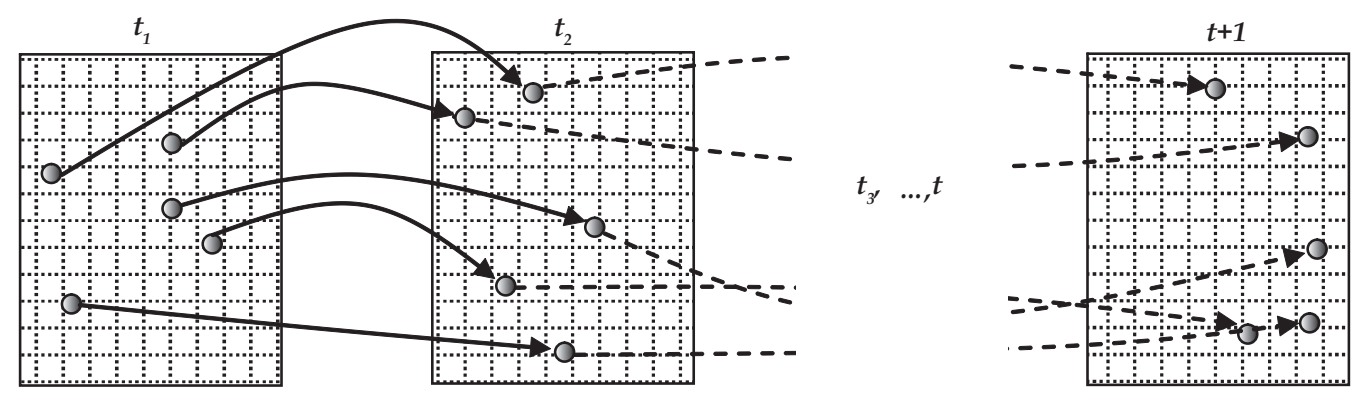

Graph 1: Spatiotemporal demand distribution and matching. 
Diachronic data for $\mathbf{T}$ time periods $(\mathrm{T}=1, \ldots, t)$ concerning demand $\left(\mathbf{W}_{\mathbf{T}}\right)$ which must be served by $\mathbf{P}$ mobile units $(\mathrm{P}=1, \ldots, n)$ are collected initially. The demand is expressed by a list of Cartesian coordinates indicating the geographical location $\left(X_{i}^{T}, Y_{i}^{T}\right)$ of each incident. The number of incidents $\mathbf{W}_{\mathbf{t}+\mathbf{1}}$, for time $\mathbf{t}+\mathbf{1}$ as well as their geographic coordinates $\left(X_{i}^{t+1}, Y_{i}^{t+1}\right)$ are then determined.

For the definition of their estimated geographical coordinates, spatiotemporal series of events are created fictitiously expressing incident movement over time. The prediction is, thus, based on the hypothesis that incidents that occur in any period are connected with incidents of the next with a one-to-one relation. Although normally each time $\mathbf{T}$ does not contain the same number of incidents $\mathbf{W}_{\mathbf{T}}$, with every other, the entire set of incidents should be correlated throughout time $\mathbf{T}=1$ to time $\mathbf{T}=t$.

This correspondence is based on the distance $D_{i o}^{T}$ of each incident i $\left(X_{i}, Y_{i}\right)$ from reference point $O\left(X_{0}, Y_{0}\right)$ for two successive time periods or time interval $\mathbf{T}$, and is expressed according to eqn (5).

$$
D_{i o}^{T}=\sqrt{\left(X_{i}-X_{o}\right)^{2}+\left(Y_{i}-Y_{o}\right)^{2}} .
$$

Points are then shortened in ascending order by distance and matched so that the first point in period $t_{1}$ corresponds to the first point of the next period, $t_{2}$ and so on. The same process is followed for the remaining points, generating in this respect the neural network's input matrix. Due to the different number of events $\mathbf{W}_{\mathbf{T}}$ for each time $\mathbf{T}$, there is a possible issue concerning the likelihood of filling the input matrix partially and with respect to prediction potential incompletely. To deal with this problem, two different approaches can be applied, depending on the relation of incidents of previous and subsequent time period:

(a) If incidents $\mathbf{W}_{t}$ are more than predicted $\mathbf{W}_{t+1}$, then they are clustered using fuzzy logic. The number of clusters equals $\mathrm{W}_{t+1}$. Thus, the number of incidents is reduced, while maintaining their spatial correlation.

(b) If incidents $\mathbf{W}_{t}$ are less than predicted $\mathbf{W}_{t+1}$, then spatial interpolation is applied so that the number of incidents is incremented to the predicted value, as this is expressed in eqns (6) and (7).

$$
\begin{gathered}
X_{i t}=\frac{X_{i t+1}+X_{i t-1}}{2} . \\
Y_{i t}=\frac{Y_{i t+1}+Y_{i t-1}}{2} .
\end{gathered}
$$

In the case where the matrix remains partially completed, it is divided into the same number of parts as the number of consecutive parts of the table. To proceed according to the proposed approach and predict incident locations, a different neural network is created for each new matrix.

Finally, the predicted points representing the approximate location of events are clustered through the utilisation of the Fuzzy C-means algorithm with the centres of each cluster playing the role of a fire engine's location. Under these circumstances and in terms of the locational planning aspect of the problem, cluster points represent demand nodes, while cluster centres correspond to the optimal facility locations.

\section{CASE STUDY: EMERGENCY MANAGEMENT IN ATHENS, GREECE}

In this section, we apply the derived model for the location of five fire confronting vehicles in the Metropolitan Area of Athens, Greece. Aiming to support the decision-making process according to real data in terms of both emergency management and response, we use records of incidents that 
were recorded and operationally served by the Athens Fire Department during the first 11 months of 2008. The dataset provided by the Athens Fire Department included information on the type, the geographic location and time stamp for each event.

According to the proposed methodology our aim is to analyse and predict the number of events. A parallel goal is the locational-spatial approximation of the events that will, according to trends and tendencies, occur in the 12th month of 2008. With the beforehand knowledge of incidents' locations, the service can organise the location of five mobile units in the city, so that assistance can be offered in the shortest and most efficient way. To further evaluate the above process with respect to its accuracy, the produced results were cartographically compared to the location of the real incidents of the specific month.

A multilayer perceptron (MLP) neural network is employed to first, predict the number of incidents. MLP is a hierarchical structure of several perceptrons that learns nonlinear function mappings and overcomes the shortcomings of these single-layer networks. The multilayer perceptron is capable of learning a rich variety of nonlinear decision surfaces. Nonlinear functions are represented by multilayer perceptrons with units that use nonlinear activation functions.

After the processes of training and testing were completed, the selected network identified and decoded relations among the input data that referred to the first 11 months of 2008, with a linear correlation coefficient $r=0.97$. On the following step and to check whether further calibration of the model was needed, the number of incidents for the 12th month of 2008 (test data) was predicted and calculated as 56 , which compared to the corresponding number of 58 events differs only slightly (3.4\%), and thus no modification of its functional parameters was needed. (Table 1).

After the prediction of the number of events is completed, the process of approximating the geographical location of each incident follows. To this end, the required time series or spatiotemporal matrix of events is created. Table 2 shows a partial extract of the specific database, containing information about each separate event concerning its unitary id, distances from reference point $\mathrm{O}$ and the X, Y Cartesian coordinates of six points for three successive time periods. To spatially relate and match counterpart events, their Cartesian coordinates and $D_{i o}^{T}$ distances are sorted and thus

Table 1: Number or incidents per month.

\begin{tabular}{lc}
\hline Month & Number of incidents \\
\hline January & 47 \\
February & 46 \\
March & 45 \\
April & 51 \\
May & 42 \\
June & 62 \\
July & 60 \\
August & 40 \\
September & 62 \\
October & 56 \\
November & 53 \\
December & 58 \\
Neural net output & 56 \\
\hline
\end{tabular}


Table 2: Event locations per month (demand).

\begin{tabular}{rccrcccccccc}
\hline$t_{1}$ & Distance & $\mathrm{X}$ & $\mathrm{Y}$ & $t_{2}$ & Distance & $\mathrm{X}$ & $\mathrm{Y}$ & $t_{3}$ & Distance & $\mathrm{X}$ & $\mathrm{Y}$ \\
\hline 1 & 5075 & 4449 & 2442 & 1 & 5926 & 3245 & 4958 & 1 & 5925 & 5667 & 1730 \\
2 & 4268 & 3652 & 2208 & 2 & 6273 & 5347 & 3280 & 2 & 4538 & 3521 & 2863 \\
3 & 5874 & 5611 & 1738 & 3 & 5909 & 5902 & 300 & 3 & 4926 & 4901 & 498 \\
4 & 6098 & 6055 & 729 & 4 & 6301 & 4654 & 4248 & 4 & 5861 & 5080 & 2923 \\
5 & 5724 & 4597 & 3412 & 5 & 5335 & 4649 & 2618 & 5 & 5621 & 4719 & 3054 \\
6 & 6240 & 6133 & 1147 & 6 & 5722 & 5513 & 1533 & 6 & 5860 & 5611 & 1690 \\
\hline
\end{tabular}

Table 3: Matrix of interconnected events.

\begin{tabular}{rccrcccccccr}
\hline$t_{1}$ & Distance & $\mathrm{X}$ & $\mathrm{Y}$ & $t_{2}$ & Distance & $\mathrm{X}$ & $\mathrm{Y}$ & $t_{3} \mathbf{p}$ & Distance & $\mathrm{X}$ & $\mathrm{Y}$ \\
\hline 2 & 4268 & 3652 & 2208 & 5 & 5335 & 4649 & 2618 & 2 & 4538 & 3521 & 2863 \\
1 & 5075 & 4449 & 2442 & 6 & 5722 & 5513 & 1533 & 3 & 4926 & 4901 & 498 \\
5 & 5724 & 4597 & 3412 & 3 & 5909 & 5902 & 300 & 5 & 5621 & 4719 & 3054 \\
3 & 5874 & 5611 & 1738 & 1 & 5926 & 3245 & 4958 & 6 & 5860 & 5611 & 1690 \\
4 & 6098 & 6055 & 729 & 2 & 6273 & 5347 & 3280 & 4 & 5861 & 5080 & 2923 \\
6 & 6240 & 6133 & 1147 & 4 & 6301 & 4654 & 4248 & 1 & 5925 & 5667 & 1730 \\
\hline
\end{tabular}

Table 3 is created. In the specific table, each row represents a fictitious spatiotemporal line of incident movement, which substantiates the methodologically hypothesised evolution process.

As stated earlier, for the months with more incidents than predicted, the referencing points are grouped by means of fuzzy logic into 56 clusters. For the months with fewer than 56 incidents, additional points are generated via spatial interpolation of points from previous and next time periods. Finally, when the number of points is the same for each time period, the input matrices are fed to the MLP neural network model and the prediction results in terms of the approximated locations of points that are shown in Map 1 jointly with the pragmatic ones.

During the final stage of the process, the five fire engines are optimally sited utilising the Fuzzy C-means algorithm. It should be noted that the algorithm is applied both to the prognosed and the recorded datasets of incidents, and in this respect, two different solution schemes are generated and displayed in Map 1, defining in this respect, the prediction and the pragmatic centres.

\subsection{Evaluation of results and discussion}

To verify from a geostatistic point of view that the results were legitimate and thus acceptable, four embedded buffers were created in a GIS environment, incremented by $300 \mathrm{~m}$ around each real incident that was archived for the 12th month of the studied year (Map 2).

As a measure of spatial accuracy we utilise the percentage of the predicted points that fall within of each of the three incrementing buffer zones generated around actual events calculating in this respect the spatial deviation of the prognosis. In sum, $50.8 \%$ of the predicted points lay within a range of $300 \mathrm{~m}$ of the actual incidents, while $74.2 \%$ of them lay within a range of $600 \mathrm{~m}$. It should be stressed, however, that incidents located more than $600 \mathrm{~m}$ away from the real incidents, 


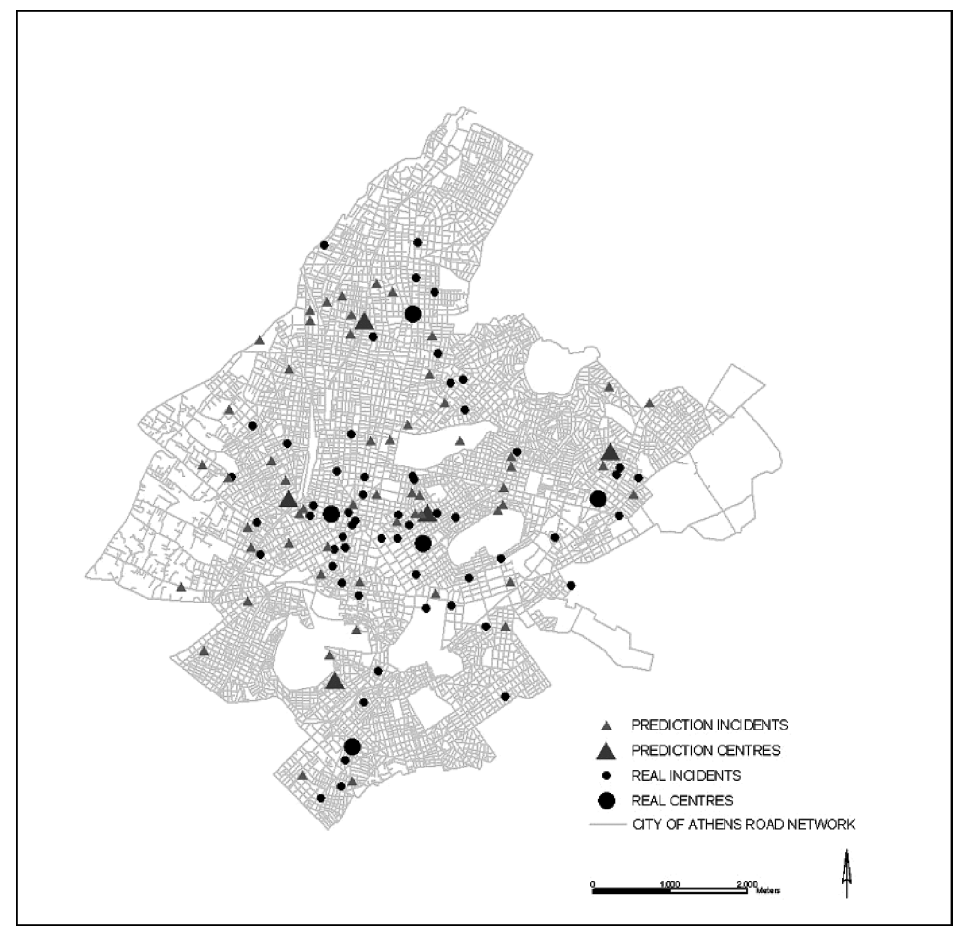

Map 1: Pragmatic and prognosed supply and demand.

exhibit a westward shift (Table 4, Graph 2) which can be accredited to the geometric idiom of the study area.

Subsequently and to investigate whether both distribution reflect the same spatial pattern, the nearest neighbour index $\mathrm{R}$ was calculated for both predicted and actual sets. Additionally, the corresponding $\mathrm{Z}$ value was also defined, clarifying in this respect whether or not the $\mathrm{H}_{0}$ hypothesis that the distributions statistically coincide with the normal distribution, is true.

The performed statistical analysis of the real incidents occurring in the 12th month indicated that $\mathrm{R}=0.826$ and $\mathrm{Z}=-2.45$. The results, therefore, display a tendency towards clustering, since $\mathrm{R}<1$. The zero case hypothesis $\mathrm{H}_{0}$ has to be rejected, since the value for the normal distribution for a level of confidence of $95 \%$ is \pm 1.96 , which is greater than the value of -2.45 for $Z$ that was observed. The corresponding values for the predicted results are $\mathrm{R}=0.72$ and $\mathrm{Z}=-3.82$. This implies a clustered spatial pattern with the null hypothesis to be rejected, since -3.82 is less than \pm 1.96 . The nearest neighbour analysis is also applied to the two sets of five service centres. According to these, the pattern of the real centres for the optimal confrontation of the events of the 12th month is dispersed, with $R=1.50$ and $Z=2.132$. The same resulted for the spatial distribution of the predicted centres with $\mathrm{R}=1.63$ and $\mathrm{Z}=2.706$, which also implies a dispersed pattern.

Summarising the findings of the evaluation stage of the process, it can be argued that the designed and applied neural network model succeeded in the task of analysing and predicting the spatial pattern of demand for the specific phenomenon. It can also be stated that in the case where a greater volume of diachronic data might be available, the output would be significantly more accurate and the observed westward shifting of the predicted points scatter diagram would be minimised. This 


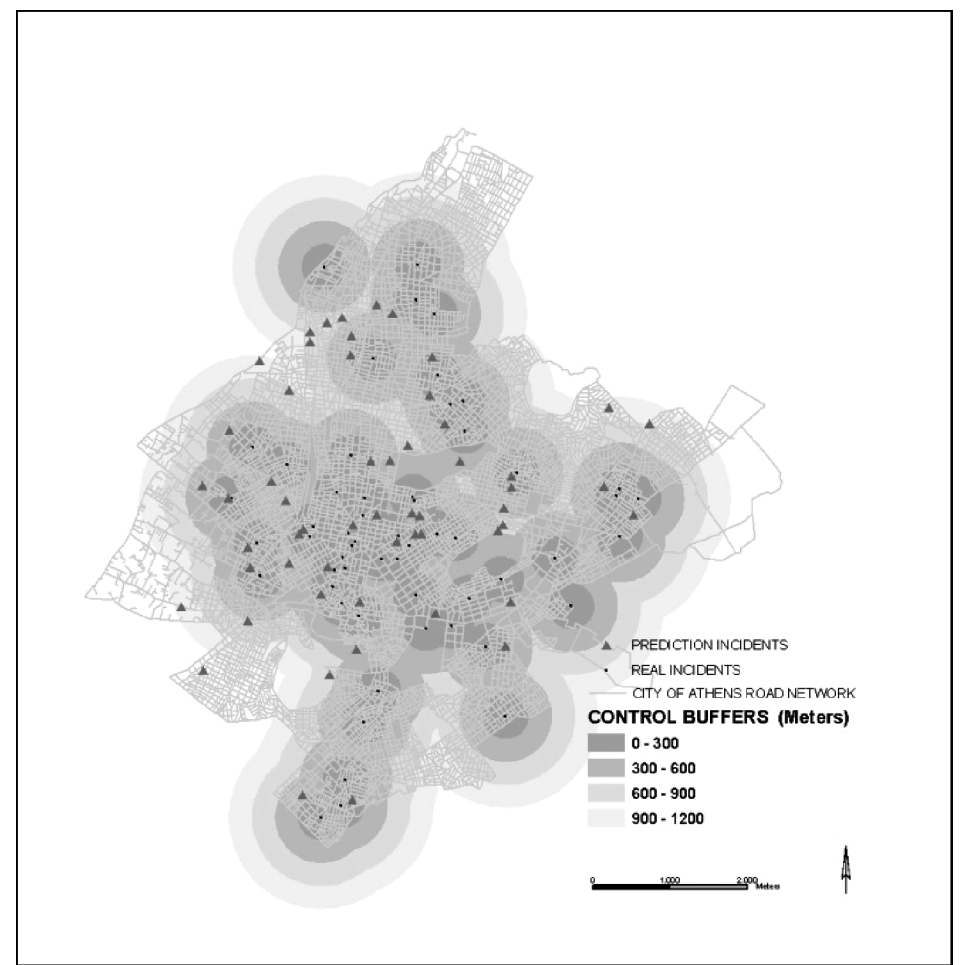

Map 2: Embedded buffers of $300 \mathrm{~m}$ around pragmatic events.

Table 4: Amount of coverage by control buffer.

\begin{tabular}{lc}
\hline Buffers & Coverage \% \\
\hline 300 & 50.8 \\
600 & 74.2 \\
900 & 91.7 \\
1200 & 97.3 \\
\hline
\end{tabular}

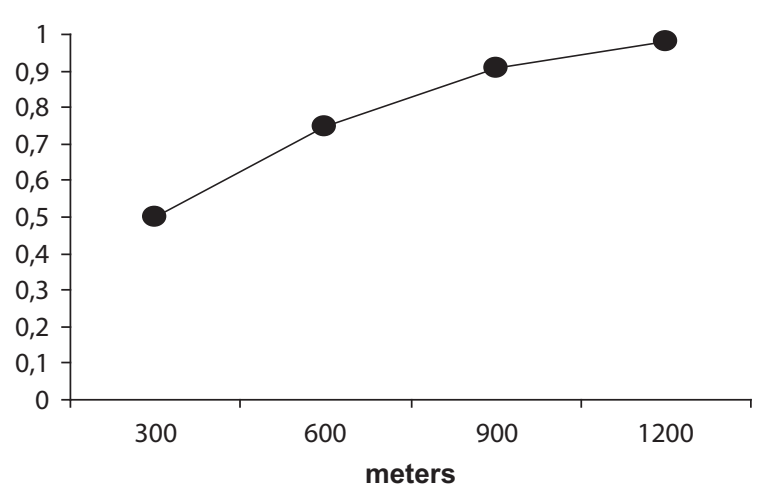

Graph 2: Percentage of coverage by control buffer. 
fact, however, is largely related to data availability and occurrence frequency regarding the incidents of interest in the respective time and place.

\section{CONCLUDING REMARKS}

In this paper, we introduce a methodological framework for spatiotemporal point pattern analysis based on artificial intelligence methods, such as neural networks and fuzzy logic, to predict evolvement and distribution of demand and, respectively, define the optimal facilities sites, in stochastic location-allocation problems. The problem-solving process is divided in three succeeding stages which first, provide corresponding capabilities for diachronic analysis of the spatial pattern of demand, second, predict its future evolution, in terms of both size and geographic locations and finally, define optimal sites for supply centres as well as allocate demand to them. Neural networks and fuzzy logic can make a positive contribution to geographical analysis because they offer flexibility, create models without the need for predefined rules, do not make assumptions about the nature and interrelatedness of the data, are simple from a statistical point of view and provide the ability to learn from data. They are also capable of recognising patterns among diachronic data, the volume, quality and consistency of which is of critical importance since it greatly affects the robustness and functionality of the resulting network. Insufficient or limited amount of data can lead to learning process to confine to local minima, failing to identify relations and interactions among original data.

The location of $\mathbf{P}$ service centres follow based on the theory of fuzzy sets, via the implementation of the modified algorithm Fuzzy C-means. The overall approach confirms the mainstream tendency, according to which applications of artificial intelligence methods in the resolution of spatial problems provides the possibility of tracking and decoding relations that are not beforehand visible and comprehensible. This possibility is of decisive importance, especially when human life is one of the most important parameters of the investigated problem, as in the case study presented above concerning the location of fire brigade vehicles in the Metropolitan area of Athens.

An aspect of the present work that should be examined further is the points' correspondence process which is directly associated with the precision of the predicted pattern. In this paper, the correspondence is based on the distance of each point from the Cartesian origin of the reference coordinate system and the final results are considered satisfactory. With the definition and application of alternative matching rules and processes, such as the generation of sequential sets of points that belong to different time periods based on their minimum observed spatiotemporal distance and the refinement of the concurrent management of the differing size of the available datasets, the proposed approach is expected to be improved significantly.

\section{REFERENCES}

[1] Photis Y.N., Koutsopoulos K., Supporting Locational Decision Making: regionalization of Service Delivery systems, Studies in Regional \& Urban Planning 1, pp. 13-34, 1994.

[2] Daskin M., Network and Discrete Location, J. Wiley \& Sons: New York, 1995. doi: http:// dx.doi.org/10.1002/9781118032343

[3] Jia H., Ordonez F., Dessouky M., A modeling framework for facility location of medical services for large-scale emergencies, IIE Transactions, 39(1), 2011.

[4] Garfinkel S., Church L., Locating an obnoxious facility on a network, Transportation Science 12/2(107), pp.107-118, 1978.

[5] Haykin, S., Neural Networks: A Comprehensive Foundation, Macmillan: New York, 1994.

[6] Guerrero F., Lozano S., Onieva L., Larraneta J., Kohonen maps for solving a class of location allocation problems, European Journal of Operational Research 108, 106-117, 1998. doi: http://dx.doi.org/10.1016/S0377-2217(97)00046-5 
[7] Wilson D., Corcoran J., Lews M., Data clustering and rule abduction to facilitate crime hot spot prediction, Fuzzy Days 2001, LNCS 2206, Springer Verlag, pp. 807-821, 2001.

[8] Houck R., Joines A., Kay G, Comparison of genetic algorithms, random restart and two optswitching for solving large location allocation problems, Computers and Operations Research 23, pp. 587-596, 1996. doi: http://dx.doi.org/10.1016/0305-0548(95)00063-1

[9] Gen M., Gong D., Yamazaki G., Xu W., Hybrid evolutionary methods for capacitated location allocation problem, Computers and Industrial Engineering, 33, Elsevier Science Ltd, pp. 577-580, 1997.

[10] Gen M., Zhou G., Min H., The balanced allocation of customers to multiple distribution centers in the supply chain network: a genetic algorithm approach, Computers and Industrial Engineering, 43, Elsevier Science Ltd, pp. 251-261, 2002.

[11] Burrough P., Development of intelligent GIS. International Journal of Geographical Information Systems 6(1), pp. 1-11, 1992. doi: http://dx.doi.org/10.1080/02693799208901891

[12] Leung Y., Leung K.S., An intelligent expert system shell for knowledge-based GIS: 1 The tools, 2 Some applications. International Journal of Geographic Information Systems 7, pp. 189-213, 1993. doi: http://dx.doi.org/10.1080/02693799308901951

[13] Abel J., Davis R., The systems integration problem. International Journal of Geographic Information Systems 8, pp. 1-12, 1994. doi: http://dx.doi.org/10.1080/02693799408901984

[14] Wena M., Iwamura K., Facility location-allocation problem in random fuzzy environment: Using $(\alpha, \beta)$-cost minimization model under the Hurewicz criterion, Computers \& Mathematics with Applications, 55, pp. 4, 2008

[15] Meilin W., Iwamurab K., Fuzzy facility location-allocation problem under the Hurwicz criterion, European Journal of Operational Research, 184 (2), pp. 16, 2008.

[16] Densham, P.J. \& G. Rushton., Decision Support systems for Locational Planning. In. Behavioural Modelling in Geography, eds R.G. Golledge \& H.Timmermans. Croom Helm: New York, 1987.

[17] Muralia P., Ordonez http://www.sciencedirect.com/science/article/pii/S0038012111000450 aff2 F., Dessouky http://www.sciencedirect.com/science/article/pii/S0038012111000450 aff2 M., Facility location under demand uncertainty: Response to a large-scale bio-terror attack, Socio-Economic Planning Sciences, in press, 2011.

[18] Huang Y., Fan Y., Modeling Uncertainties in Emergency Service Resource Allocation, Journal of Infrastructure Systems, 17(35), 2011.

[19] Beraldi P., Bruni M.E., A probabilistic model applied to emergency service vehicle location, European Journal of Operational Research, 196, pp. 1, 2009. doi: http://dx.doi.org/10.1016/j. ejor.2008.02.027

[20] Harper P.R., Shahani A.K., Gallagher J.E., Bowie C., Planning health services with explicit geographical considerations: a stochastic location-allocation approach, Omega, 33, pp. 2, 2005.

[21] Dacey M.F., Two dimensional random point patterns. A review and interpretation, Papers in Regional Science, 13(1), 1964. doi: http://dx.doi.org/10.1007/BF01942559

[22] Rogers, A., Quadrat Analysis of Urban Dispersion: I. Theoretical Techniques, Environment and Plannining, 1, pp 47-80, 1969. doi: http://dx.doi.org/10.1068/a010047

[23] Photis Y.N., Tsobanoglou S., Measuring urban dynamics through public and private sector concentration patterns. The case of Thessaly, Greece, in The Contexts, Dynamics and Planning of Urban Development, eds Y. Psycharis \& P. Skayannis, University of Thessaly Publications: Volos, 2009.

[24] Clark, P.J., Evans F.C., Distance to nearest neighbor as a measure of spatial relationships in populations, Ecology, 35(4), pp. 445-453, 1954. doi: http://dx.doi.org/10.2307/1931034 
[25] Manetos P., Photis Y.N., Simulation of urban system evolution in a synergetic modelling framework. The case of Attica, Greece, 43rd European Congress of the European Regional Science Association, Jyvaskyla: Finland, 2003.

[26] Openshaw S., Openshaw C., Artificial Intelligence in Geography, John Wiley \& Sons Ltd.: England, 1997.

[27] Ripley B.D., Pattern recognition and neural networks, Cambridge University Press, New York, 2008.

[28] Zadeh. L.A., Fuzzy Sets, Information Control, 8, pp. 338-353, 1965. doi: http://dx.doi. org/10.1016/S0019-9958(65)90241-X

[29] McNeill, D., Freiberger, P., Fuzzy Logic, Simon and Schuster: New York, NY, 1994.

[30] Pereza J.A.M., Vegaa J.M.M., Verdegay J.L., Fuzzy location problems on networks, Fuzzy Sets and Systems, 142(3), pp. 393-405, 2004. doi: http://dx.doi.org/10.1016/S0165-0114(03)00091-5 